\title{
Optical properties of silicon nitride films deposited by hot filament chemical vapor deposition
}

\author{
Sadanand V. Deshpande ${ }^{\text {a) }}$ and Erdogan Gulari \\ Department of Chemical Engineering and Center for Display Technology and Manufacturing, \\ The University of Michigan. Ann Arbor, Michigan 48109 \\ Steven W. Brown ${ }^{\text {b) }}$ and Stephen C. Rand \\ Applied Physics Program, The University of Michigan, Ann Arbor, Michigan 48109
}

(Received 16 January 1995; accepted for publication 13 March 1995)

\begin{abstract}
Silicon nitride films were deposited at low temperatures $\left(245-370^{\circ} \mathrm{C}\right)$ and high deposition rates (500-1700 $\AA / \mathrm{min}$ ) by hot filament assisted chemical vapor deposition (HFCVD). Optical properties of these amorphous silicon nitride thin films have been extensively characterized by absorption, photoluminescence (PL), photoluminescence excitation, and electroluminescence measurements. The optical band gap of the films was varied between 2.43 and $4.74 \mathrm{eV}$ by adjusting the flow rate of the disilane source gas. Three broad peaks at 1.8, 2.4, and 3.0 eV were observed in the PL spectra from these films. A simple qualitative model based on nitrogen and silicon dangling bonds adequately explains the observed PL features. The photoluminescence intensity observed in these films was 8-10 times stronger than films deposited by plasma enhanced chemical vapor deposition, under similar conditions. The high deposition rates obtained by HFCVD is believed to introduce a large number of these optically active defects. (C) 1995 American Institute of Physics.
\end{abstract}

\section{INTRODUCTION}

Amorphous silicon nitride $\left(a-\mathrm{SiN}_{x}: \mathrm{H}\right)$ is extensively used in the microelectronics industry for a wide variety of applications including use as an oxidation mask, a dopant diffusion barrier, the gate dielectric in field effect and thin film transistors, an encapsulant for III- $\mathrm{V}$ semiconductors, an interlevel dielectric, a charge storage layer in MNOS nonvolatile memories, and as a final passivation layer for device packaging. Silicon nitride thin films are conventionally deposited at high substrate temperatures $\left(700-900^{\circ} \mathrm{C}\right.$ ) by thermal chemical vapor deposition (CVD) and at lower temperatures $\left(200-400^{\circ} \mathrm{C}\right)$ using plasma methods. ${ }^{1,2}$ Low temperature $\left(25-400^{\circ} \mathrm{C}\right)$ deposition schemes have become particularly important due to the increasing complexity of semiconductor processing and a need for thermal budgeting. Several different low-temperature deposition methods have been employed in the past for silicon nitride film growth, including plasma-enhanced CVD techniques ${ }^{2-7}$ and sputtering., 8

While previous studies of silicon nitride films focused primarily on the electronic and paramagnetic properties of the material, several studies of the optical properties of thermal and plasma enhanced chemical vapor deposition (PECVD) silicon nitride films have been made, including photoluminescence (PL), ${ }^{9-12}$ photoluminescence excitation (PLE) ${ }^{13-15}$ and luminescence decay ${ }^{16}$ studies. In this work, the optical properties of hot filament-assisted chemical vapor deposition (HFCVD) $a-\mathrm{SiN}_{x}: \mathrm{H}$ films deposited under varying conditions are discussed. In the following section, experimental techniques used in this work are presented. Significant experimental results are presented in Sec. III,

\footnotetext{
Author to whom all correspondence should be addressed.

b) Present address: Naval Research Laboratory, Washington, DC 20375-5320.
}

followed by a discussion of these results in Sec. IV. Finally, in Sec. V, the conclusions are summarized.

\section{EXPERIMENT}

\section{A. Film deposition}

Silicon nitride films studied in this work were grown by HFCVD. In HFCVD, reactant gases are flowed over a heated filament to form precursor species which subsequently impinge on the substrate surface, resulting in the deposition of high quality films. The general applicability of the technique is illustrated by the fact that HFCVD has been successfully used to grow a wide variety of films, including diamond, ${ }^{17-19}$ boron nitride, ${ }^{20}$ aluminum nitride, ${ }^{21}$ titanium nitride, ${ }^{22}$ and boron carbide, ${ }^{23}$ as well as amorphous silicon nitride. ${ }^{24,25}$

The reactor system employed for the deposition of silicon nitride thin films studied in this work has been described previously. ${ }^{26,27}$ In this system, ammonia was flowed over a heated tungsten filament while disilane was introduced into the reactor between the tungsten filament and the substrate by a gas dispersal ring designed to promote uniform film growth. Films were deposited on quartz and silicon substrates under a variety of deposition conditions, as summarized in Table $\mathrm{I}$.

The physical properties of HFCVD silicon nitride films have been extensively characterized. Previous studies include $\mathrm{x}$-ray photoelectron spectroscopy (XPS) measurements which determined the stoichiometry of the films as a function of deposition conditions and spectroscopic ellipsometry measurements of the refractive indices of the films. ${ }^{27}$ Because of the large effect of hydrogen incorporation on properties such as etch rates in these films, the hydrogen content in HFCVD films was measured previously by both fourier-transform infrared absorption (FTIR) and elastic recoil detection (ERD) techniques. ${ }^{28}$ Experimental techniques used in the analysis of $a-\mathrm{SiN}_{x}: \mathrm{H}$ films discussed in this work are described below. 
TABLE I. Deposition parameters for $a-\operatorname{SiN}_{x}: H$ thin films.

\begin{tabular}{cc}
\hline parameter & Set point(s) \\
\hline Reactor pressure & $0.5 \mathrm{Torr}$ \\
Substrate temperature & $245-370^{\circ} \mathrm{C}$ \\
Filament temperature & $1500-1850^{\circ} \mathrm{C}$ \\
Ammonia flow rate & $80 \mathrm{sccm}$ \\
Disilane flow rate & $1.1-3.2 \mathrm{secm}$ \\
Hydrogen flow rate & $0-230 \mathrm{sccm}$ \\
Argon flow rate & $0-142 \mathrm{sccm}$ \\
\hline \hline
\end{tabular}

\section{B. Electron spin resonance}

Electron spin resonance (ESR) experiments were performed with a Varian Associates model E-112 spectrometer. For an unpaired electron with spin $1 / 2$, allowed transitions occur for $\Delta E=h v=g \beta H$, where $h$ is Planck's constant, $v$ the frequency of the microwave radiation, $H$ the magnetic field strength, and $\beta$ the magnetic moment. The free electron $g$ value is 2.0023 , though, in general, the value of $g$ depends on the identity of the particle and the nature of its chemical environment. The spectrometer was operated at a nominal frequency of $9.23 \mathrm{GHz}$ ( $X$ band), giving a transition at an applied magnetic field of $3300 \mathrm{G}$ for an electron $g$ value of 2.0023 .

The static magnetic field was generated by a water cooled electromagnet regulated to $3.3 \mathrm{mG}$ ripple at $3300 \mathrm{G}$. The air gap was $3 \mathrm{in}$. wide and the pole caps were of the ring-shim configuration, ensuring a field uniformity of \pm 15 mG over the sample volume. Microwave radiation was generated by a klystron source and was coupled via a gold plated waveguide to a $\mathrm{TEM}_{11}$ resonant cavity. The frequency of the klystron was stabilized using an automatic frequency controller and locked to the peak of the coupling efficiency of the cavity. A single chumber cavity was used with a quartz sample holder held midway between the pole pieces of the magnet. Care was taken to ensure that the signal was not saturated at the microwave power level used for the measurements $(0.2 \mathrm{~mW})$. Measured spin densities were calibrated against a weak pitch reference with a strength of $10^{13}$ spins $/ \mathrm{cm}^{3}$, giving an estimated accuracy of the measured densities to be within a factor of 2 for this single cavity configuration.

\section{Absorption}

The optical (Tauc) band gap of the silicon nitride films was determined by depositing films on UV-grade quartz substrates and measuring the absorption with a Cary UV-Visible spectrophotometer. The optical band gap was determined from the expression given by Tauc for amorphous materials: ${ }^{29}$

$$
\hbar\left(\omega \alpha(\omega)=\left(\hbar \omega-E_{\mathrm{g}}^{(p)}\right)^{2},\right.
$$

where $\hbar$ is Planck's constant, $\omega$ the frequency, and $E_{g}^{\text {opt }}$ the optical band gap. Therefore, by plotting $(\hbar \omega \alpha)^{0.5}$ against the photon energy $(h \omega)$, the optical band gap can be determined.

\section{Photoluminescence}

Photoluminescence (PL) experiments were performed in a standard backscatter geometry using an ISA THR 1000 spectrometer with $5 \AA$ resolution. The PL was excited with $100 \mathrm{~mW}$ of $457.9 \mathrm{~nm}$ radiation from an Argon ion laser. All lenses were UV grade fused silica, with greater than $90 \%$ transmission at wavelengths as low as $250 \mathrm{~nm}$. A grating blazed from 300 to $600 \mathrm{~nm}$ was used for spectral analysis. The signal was detected with a cooled RCA C31034a-02 photomultiplier tube (PMT) operated in photon counting mode. The PMT window was UV grade fused silica with greater than $80 \%$ transmission from 800 down to $200 \mathrm{~nm}$. The data were not corrected for the spectral response of the system.

Samples were mounted with indium foil onto a copper block attached to the cold finger of an APD Cryogenics "Heli-Trans" Model LT-3-110A open-cycle refrigerator for temperature-dependent PL measurements. The sample temperature was monitored by a GaAs diode mounted on top of the copper block and regulated to within $0.2 \mathrm{~K}$ from 77 to $300 \mathrm{~K}$ by a Scientific Instruments controller capable of providing up to $20 \mathrm{~W}$ of heater power. $\Lambda$ turbomolecular pump (Balzers) maintained the dewar system at a pressure $2 \times 10^{-6}$ Torr or less for all low-temperature measurements to minimize condensation on the sample. Dewar windows were also UV fused silica.

\section{E. Photoluminescence excitation}

In photoluminescence excitation (PLE), the dependence of luminescence intensity at a fixed wavelength is measured as a function of the excitation energy. This measurement can provide additional information concerning energy levels involved in the observed luminescence. The dependence of the luminescence intensity was therefore measured at a fixed emission energy of $2.25 \mathrm{eV}$ for excitation energies in the range from 1.9 to $5.0 \mathrm{eV}$. PLE measurements were performed on these silicon nitride films using a SPEX Fluorolog ${ }^{\mathrm{TM}}$ spectrophotometer equipped with a xenon arc lamp. The luminescence signal was corrected for the spectral variation of the lamp intensity. The lamp reference spectrum was recorded by exciting a liquid dye (quantum efficiency $=1.0$ ) whose near-infrared emission was subsequently detected by a silicon photodiode.

\section{F. Luminescence decay}

Fast photoluminescence decay rates were determined using the time-correlated single-photon counting technique. All decay rates were measured at room temperature. Samples were excited with $125 \mathrm{ps}$ pulses at wavelength of $532 \mathrm{~nm}$ from a frequency-doubled, mode-locked $\mathrm{Nd}$ :YAG laser operating at a repetition rate of $76 \mathrm{MHz}$. The luminescence was imaged onto the slits of an Instruments SA HR640 double subtractive monochromator to compensate for dispersion and detected with a Hamamatsu R928 photomultiplier tube with adjustable dynode voltages. The photomultiplier tube was housed in a cooled products for research TE177RF housing providing a temperature of $40^{\circ} \mathrm{C}$ below ambient; the dark counts were thus negligible. The signal was amplified by an 
TABLE ח. Effect of disilane flow rate on the optical properties of $a-\mathrm{SiN}_{x}: \mathrm{H}$ films. PL was ercited by the 457.9 $\mathrm{nm}(2.71 \mathrm{eV})$ line of $\mathrm{Ar}^{+}$ion laser.

\begin{tabular}{lcccc}
\hline \hline Film \# & $\begin{array}{c}\text { Disilane flow rate } \\
\text { (scen) }\end{array}$ & $\begin{array}{c}\text { Optical band gap } \\
(\mathrm{eV})\end{array}$ & $\begin{array}{c}\text { PL peak position(s) } \\
\text { (eV) }\end{array}$ & $\begin{array}{c}\text { Relative peak } \\
\text { intensity }\end{array}$ \\
\hline SQT1 & 1.1 & 4.74 & $2.1 \& 1.92$ & 0.07 \\
SQT2 & 1.6 & 4.39 & $2.04 \& 1.9$ & 0.20 \\
SQT3 & 2.1 & 4.17 & $2.06 \& 1.92$ & 0.56 \\
SQT4 & 2.7 & 3.03 & 1.86 & 0.64 \\
SQT5 & 3.2 & 2.43 & 1.76 & 1.0 \\
PECVD 1 & (Low silane) & 5.2 & 2.23 & 0.41 \\
PECVD 2 & (High silane) & 4.4 & 1.98 & 1.0 \\
\hline \hline
\end{tabular}

EG\&G Ortec VT120 fast preamplifier and fed into a EG\&G Ortec 935 quad constant fraction discriminator. The discriminator output was then passed to a EG\&G Ortec 566 timeamplitude converter. Counts were registered in a $E G \& G$ Ortec 994 dual counter and timer unit and collected in a Norland 5500 Multichannel Analyzer using a 13 ns window. The system response, on the order of $500 \mathrm{ps,} \mathrm{was} \mathrm{deconvo-}$ luted from the decay spectra and analyzed using Photon Technology International's FLDEC software.

\section{RESULTS}

\section{A. Electron spin resonance}

Electron spin resonance (ESR) measurements were performed to determine the dangling bond (unpaired electron) densities in the films. ESR signals with a $g$ value of 2.003 and a linewidth of about $12.0 \mathrm{G}$ were consistently obtained from films grown under a range of deposition conditions. This signature is characteristic of a defect called the $K$ center, which is a silicon atom back bonded to three nitrogen atoms. ${ }^{30}$ To confirm the origin of the ESR signal observed in the HFCVD films, the effect of ultra-violet (UV) illumination on the measured spin density in a film deposited at $245^{\circ} \mathrm{C}$ was studied.

In this experiment, the sample was irradiated with UV light from a $150 \mathrm{~W}$ Xenon lamp. The spin density showed a gradual increase as a function of UV irradiation, increasing from $1.65 \times 10^{18} \mathrm{~cm}^{-3}$ for the as-deposited (unirradiated) sample to $3.9 \times 10^{18} \mathrm{~cm}^{-3}$ after half an hour of illumination and finally to a total spin density of $5.5 \times 10^{18} \mathrm{~cm}^{-3}$ after 1.0 $h$ of illumination. As described in previous work, the UVinduced enhancement of the ESR signal originates from a redistribution of charge at the $K$ center due to the following reaction: ${ }^{30}$

$$
K^{+}+K^{-}=2 \quad K^{0}
$$

where $K^{+}$and $K^{-}$are the diamagnetic charged states with one less electron and one extra electron, respectively, than the charge-neutral paramagnetic $K^{0}$ center detected by ESR measurements. No evidence of additional defects was observed in the ESR signal from these films, although effects of high-temperature annealing followed by UV irradiation of these films, necessary for the observation of a nitrogen dangling bond center, were not studied. ${ }^{31}$
Electron spin densities for films deposited at substrate temperatures of $370,300,245^{\circ} \mathrm{C}$ were determined to be $1.29 \times 10^{18}, 1.3 \times 10^{18}$, and $1.65 \times 10^{18} \mathrm{~cm}^{-3}$, respectively. These values are higher (by a factor of 5-10) than reported values for films deposited by conventional PECVD, ${ }^{32}$ indicating that the number of dangling bond defects are greater in films deposited by HFCVD than in films deposited by PECVD. This is not unexpected, since HFCVD growth rates (500-1700 $\AA / \mathrm{min})$ are at least an order of magnitude higher than typical PECVD deposition rates of about $50 \mathrm{~A} / \mathrm{min}^{28}$

Measured bonded hydrogen in the films decreased from $14.6 \%$ to $6.0 \%$ as the growth temperature increased from 245 to $375^{\circ} \mathrm{C}$ while total hydrogen content decreased from $17.7 \%$ to $9.5 \%{ }^{28}$ Within experimental error, therefore, there was no observed dependence of spin densities on the hydrogen content in the films. Since hydrogen is known to passivate silicon dangling bonds in the films, it may be argued that as the substrate temperature decreased the randomness in the amorphous silicon nitride network increased (creating more dangling bonds) and therefore, in spite of the increased hydrogen in the films, the dangling bond density does not decrease appreciably.

\section{B. Absorption}

The optical band gap could be reproducibly varied by changing the disilane flow rate during the film growth. Increasing the disilane flow rate increased the silicon content (or $\mathrm{Si}$ to $\mathrm{N}$ ratio) in the films, therehy decreasing the optical band gap. The changes in optical absorption with disilane flow rate are compiled in Table $\Pi$ and shown in Fig. 1. All films in this growth series were deposited at a substrate temperature of $370^{\circ} \mathrm{C}$ and filament temperature of $1700^{\circ} \mathrm{C}$.

\section{Photoluminescence}

The $a-\mathrm{SiN}_{x}: \mathrm{H}$ films deposited by HFCVD showed strong visible photoluminescence at room temperature upon excitation by an argon ion laser. The PL intensity of the HFCVD films is at least 8-10 times stronger than that observed from PECVD films and comparable to emission observed from porous silicon, for example. As shown in Fig. 1, the PL peak energy shifted to higher energy as the optical band gap increased. In addition to shifting to higher energy with increasing nitrogen content in the films, the relative PL intensity decreased with increasing optical band gap. This is 


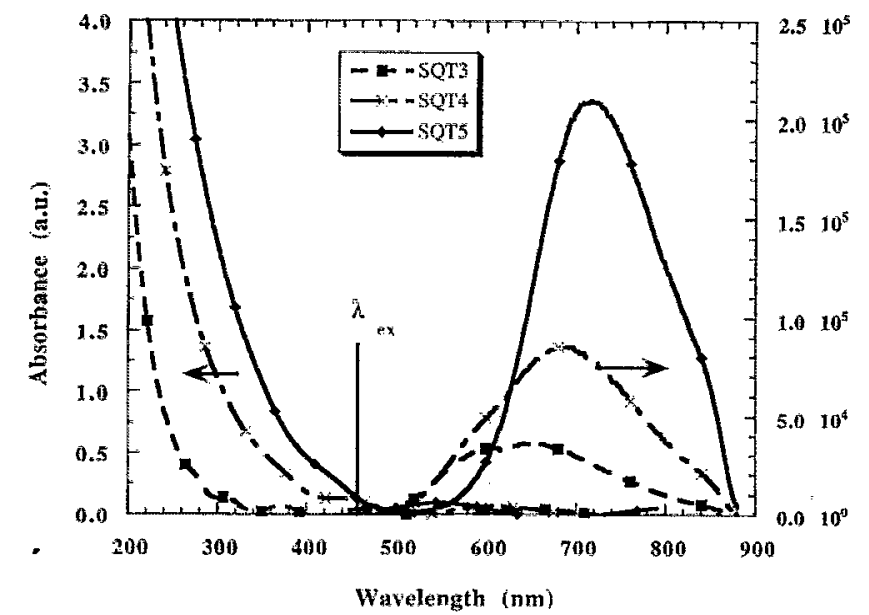

FIG. 1. PL, peak energy and intensity dependence on optical band gap.

illustrated in Table II, which shows that the emission from sample SQTl, which has an optical band gap of $4.74 \mathrm{eV}$, was $7 \%$ of the PL intensity from sample SQT5, which had an optical band gap of $2.43 \mathrm{eV}$. This may be due to the fact that there are fewer defects in the film grown at the lower disilane flow rate, that the excitation at $457.9 \mathrm{~nm}$ is not as efficient in generating luminescence from the wider band gap films, or that there are a greater number of nonradiative decay paths in films with a higher band gap. To clarify this issue, PLE measurements in the energy range from 5.0 to $1.9 \mathrm{eV}$ were performed, as discussed below.

The PL intensity showed a gradual decrease (fatigue) with time, reducing the overall PL intensity by up to $50 \%$ under long exposure times. UV-irradiation from a $150 \mathrm{~W}$ xenon lamp also reduced the overall intensity; no fatigue was seen after the films had been UV-irradiated for $1 \mathrm{~h}$ or more. Figure 2 illustrates the changes in PL intensity as a function of exposure time to $2.7 \mathrm{eV}$ radiation. The PL could be reversibly restored to its original intensity upon low-

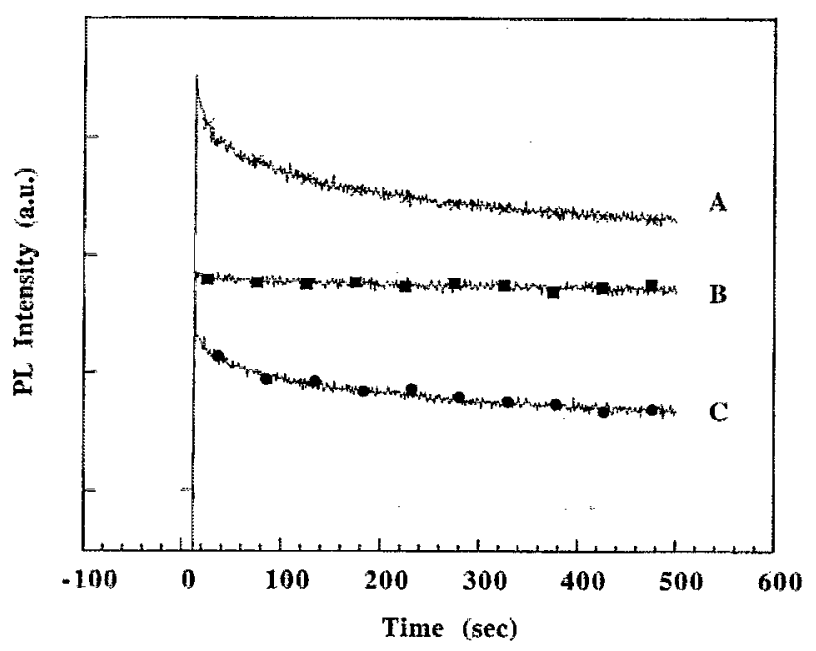

FIG. 2. PL fatigue phenomenon in HFCVD silicon nitride films: (A) PL fatigue in asedeposited film, (B) no fatigue in UV-exposed film, (C) PL fatigue after $1.0 \mathrm{~h} 800^{\circ} \mathrm{C}$ anneal.

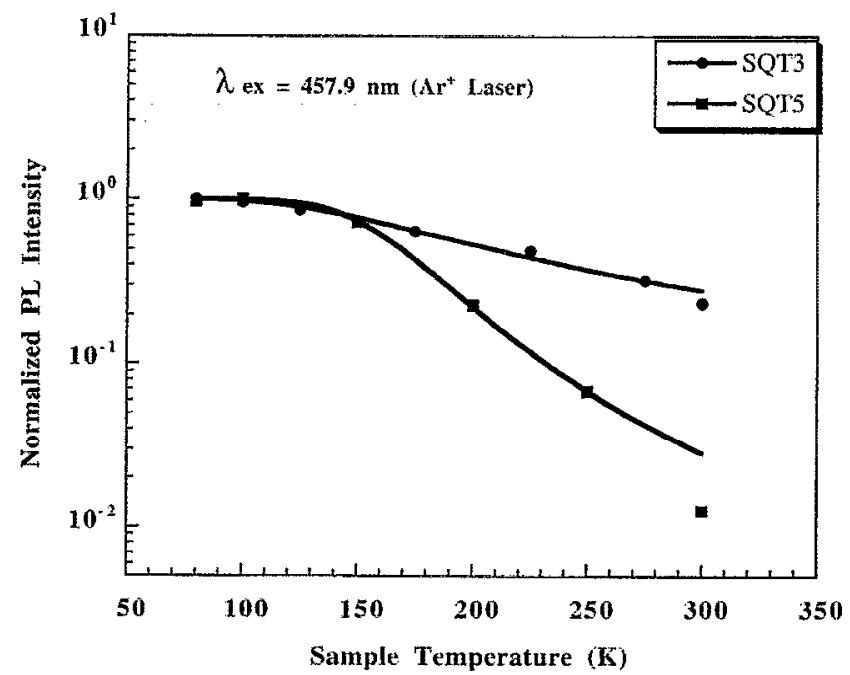

FIG. 3. Temperature dependence of photoluminescence efficiency.

temperature annealing. Our previous annealing studies ${ }^{28}$ at higher temperature showed that these defects are very stable even after $4 \mathrm{~h}$ of annealing in inert (both argon and hydrogen) atmosphere at temperatures up to $800^{\circ} \mathrm{C}$.

This fatigue phenomenon (with UV illumination -5.0 $\mathrm{eV}$ ) has been observed previously by other researchers ${ }^{3,-35}$ who found a correlation between a decrease in the PL intensity and an increase in the ESR signal upon UV-irradiation. The simultaneous decrease in PL intensity and an increase in ESR signal was attributed to the creation of additional neutral silicon dangling bond (or $K^{0}$ ) centers due to redistribution of charge between the charged diamagnetic $K$ centers $K^{+}$and $K^{-}$.

Additionally, the temperature dependence of the PL intensity (or PL efficiency) was measured in the range from 80 to $300 \mathrm{~K}$ for films SQT1, SQT3, and SQT5. As shown in Fig. 3, the PL intensities from samples SQT1 and SQT3 showed only a weak dependence on temperature, whereas the intensity varied over two orders of magnitude as the temperature increased from 80 to $300 \mathrm{~K}$ for sample SQT5. In addition, the temperature dependence of the peak position also showed very different behavior for films SQT5 and SQT3. Specifically, the peak position shifted to higher energy for sample SQT3 and to lower energy for sample SQT5 with increasing temperature. These results are in good agreement with trends observed previously by Austin el al. ${ }^{9}$ for PECVD samples with varying amounts of nitrogen.

The temperature-dependent PL data was fit using a simple theoretical model based on the thermal ionization of one carrier from an excited state to the mobility edge with a well-defined activation energy $\left(E_{A}\right):^{9}$

$$
\mathrm{PL}_{\text {efficiency }} \equiv \frac{\eta}{\eta_{0}}=\frac{1}{1+\beta \exp \left(-E_{A} / k_{B} T\right)},
$$

where, $\eta_{0}$ is the PL intensity at the lowest measured temperature $(80 \mathrm{~K})$ and $\eta$ is the PL intensity at a given sample temperature $\left(T>T_{0}\right)$. Fitting the data to Eq. (3), activation energies of 56.3 and $118 \mathrm{meV}$ were obtained for samples 


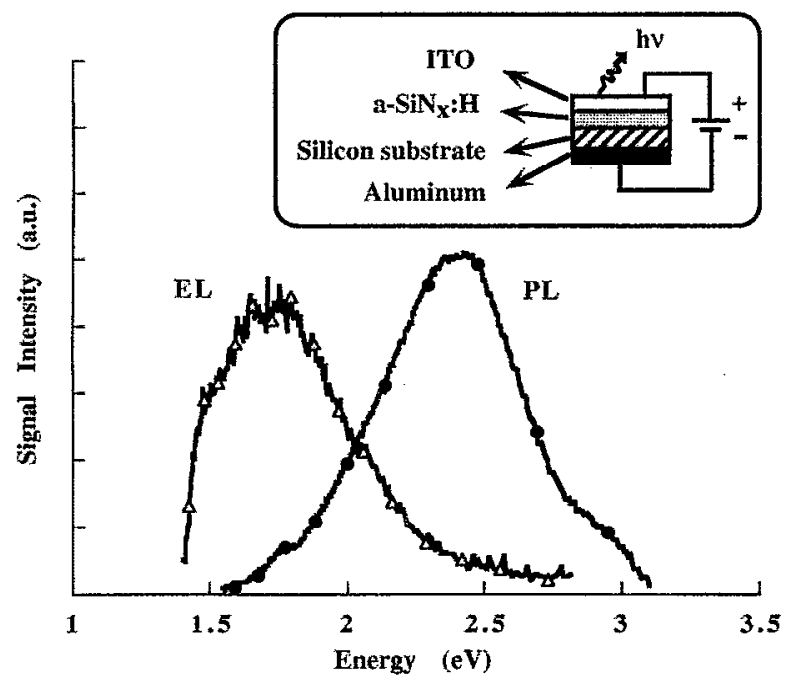

FIG. 4. EL and PL spectra for film \# SQT3. Shown in the inset is the EL device structure. Electroluminescence shows a peak at $1.76 \mathrm{eV}$ and PL was obtained with $4.14 \mathrm{eV}$ excitation.

SQT3 and SQT5 while values for the constant $\beta$ were 22.4 and 3229.0 , respectively. The value of activation energy for SQT5 is in good agreement with previous work by Austin et al. ${ }^{9}$ on PECVD films whereas the activation energy for film SQT3 is lower than that determined previously for a PECVD film of similar stoichiometry and band gap. Finally, previous work observed that the value for $\beta$ increases with decreasing nitrogen content (or increasing silicon content) in PECVD films, in agreement with results obtained in this work from HFCVD films.

The strong visible photoluminescence from HFCVD films compared to luminescence from thermal or PECVD silicon nitride films prompted us to examine the electroluminescence (EL) properties of these films. EL has been observed previously from PECVD $a-\mathrm{SiN}_{x}: \mathrm{H}$ films. For example, a recent study reported the fabrication of visible LED's employing doped amorphous silicon carbide layers for efficient electron and hole injection into the intrinsic silicon nitride active layer. ${ }^{36}$

Weak EL was observed from $a-\mathrm{SiN}_{x}: \mathrm{H}$ films using a simple device structure shown in the inset in Fig. 4. Approximately 1000-Å-thick silicon nitride films were deposited on highly doped silicon wafers (both $n$ - and $p$-type). A $3000-\AA$ thick layer of indium tin oxide (ITO) was deposited by RF sputtering on top of the $a-\mathrm{SiN}_{x}: \mathrm{H}$ film to form an $n$-type

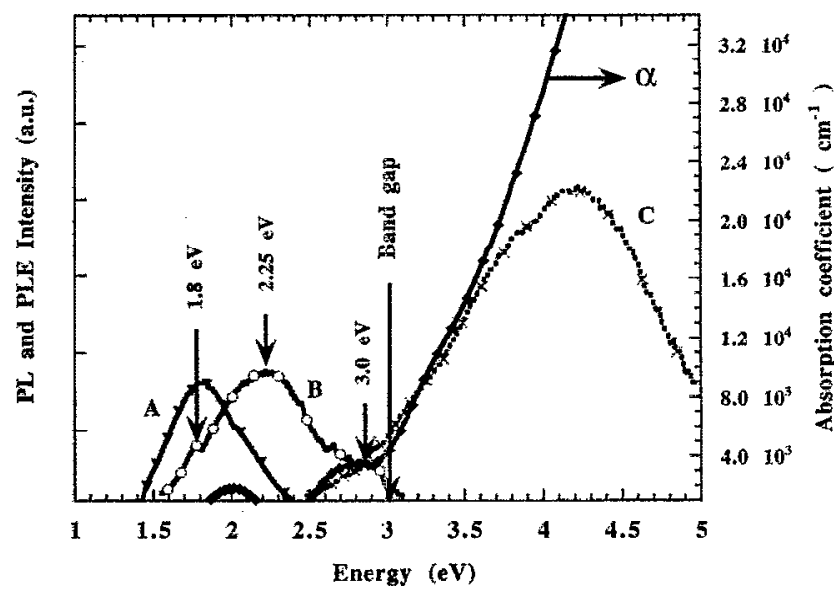

FIG. 5. PL, PLE, and absorption in Film \# SQT4: (A) PL with excitation at $2.72 \mathrm{eV},(\mathrm{B}) \mathrm{PL}$ with excitation at $4.14 \mathrm{eV}$, (C) PLE with detection at 2.25 $\mathrm{eV}$.

transparent electrode. The back-side ohmic contact was made by an 8000 - $\AA$-thick $e$-beam evaporated aluminum film which was subsequently annealed at $450^{\circ} \mathrm{C}$ in an inert atmosphere. While the electroluminescence was strong enough to be seen by eye, efficient, bright luminescent structures were not fabricated. A typical EL spectrum is shown in Fig. 4. Additionally, as shown in Fig. 4, the EL spectrum was red shifted from the PL spectrum (peak $2.41 \mathrm{eV}$ ) and the EL peak was observed at $1.76 \mathrm{eV}$.

\section{Photoluminescence excitation}

PLE studies established the existence of a second optically active state within the band gap of $a-\mathrm{SiN}_{x}$ :II films with higher nitrogen content. Figure 5 shows PL, PLE, and absorption spectra for film SQT4. As shown in the figure, the PLE spectrum closely follows the absorption edge in these films. The broad photoluminescence spectrum appears to have contribution from at least three peaks at 1.7, 2.4, and $3.0 \mathrm{eV}$. A model explaining the origin of these transitions is presented in the discussion section. The main PL peak in nitrogen-rich HFCVD films was red shifted by an average by about $425 \mathrm{mcV}$ from the PLE peak (sce Table III). There was no corresponding shift between the PL and PLE peaks for silicon-rich films, as shown in Fig. 6. It should be mentioned that the noise in the PLE spectrum for film \#SQT5 is due to lack of proper cancellation of the lamp background. In addi-

TABLE III. PL peak shift with change in excitation energy.

\begin{tabular}{lcccc}
\hline \hline Film \# & $\begin{array}{c}\text { Band gap } \\
(\mathrm{eV})\end{array}$ & $\begin{array}{c}\text { PL peak position (eV) } \\
\text { with 2.71 eV excitation }\end{array}$ & $\begin{array}{c}\text { PL peak position (eV) } \\
\text { with 4.14 eV excitation }\end{array}$ & $\begin{array}{c}\text { Shift } \\
(\mathrm{eV})\end{array}$ \\
\hline SQT1 & 4.74 & $2.08 \& 1.92$ & 2.43 & 0.43 \\
SQT2 & 4.39 & $2.04 \& 1.92$ & 2.45 & 0.47 \\
SQT3 & 4.17 & $2.06 \& 1.92$ & 2.41 & 0.42 \\
SQT4 & 3.03 & 1.86 & 2.24 & 0.38 \\
SQT5 & 2.43 & 1.76 & 1.76 & 0.0 \\
PECVD 1 & 5.3 & 2.21 & 2.65 & 0.44 \\
PFCVD 2 & 4.4 & 2.0 & 2.45 & 0.45 \\
\hline
\end{tabular}




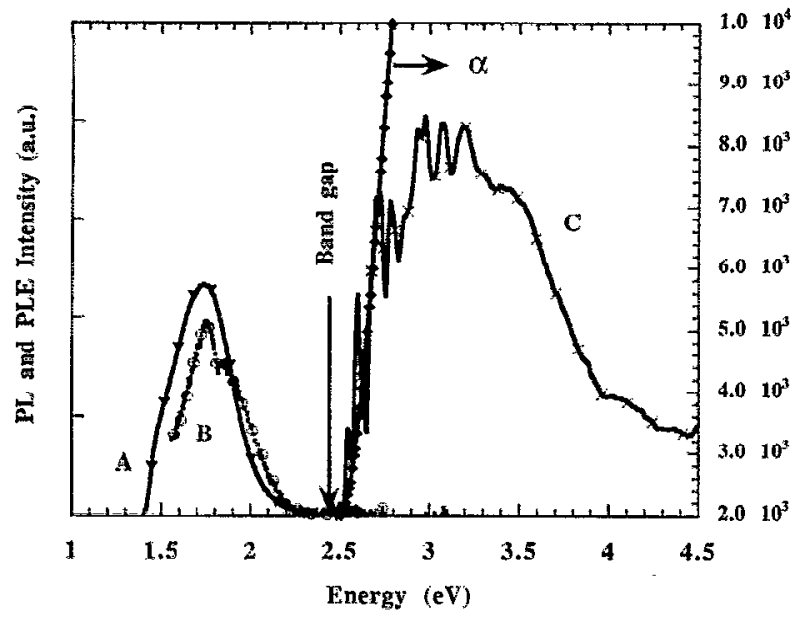

FIG. 6. PL, PLE, and absorption in Film \# SQT 5: (A) PL with excitation at $2.72 \mathrm{eV},(\mathrm{B}) \mathrm{PL}$ with excitation at $4.14 \mathrm{eV}$, (C) PLE with detection at 2.25 $\mathrm{eV}$.

tion, the PLE signal from this sample was very weak which also contributed to the background noise. The results are consistent with previous PLE studies of PECVD $a-\mathrm{SiN}_{x}: \mathrm{H}$ films ${ }^{11,15}$ and suggest that nitrogen is involved in the center responsible for the origin of the higher energy luminescence band.

\section{E. Luminescence decay}

Photoluminescence decay rates in the ns range were measured at room temperature from a series of silicon nitride films deposited with varying disilane flow rates. The decay data were fit to a linear sum of two exponentials of the form $A_{i} \exp \left(-t / \tau_{i}\right)$, where $\tau_{i}$ is the decay time constant and $A_{i}$ is a fitting parameter. The silicon nitride films exhibited a fast decay, less than $1.0 \mathrm{~ns}$, and a slower decay in the range from 4 to $10 \mathrm{~ns}$, with the contribution to the PL decay from the fast component increasing as the band gap decreased. A slight spectral dependence on the decay rates was also observed. For example, the slower decay time from sample SQT5 increased from 8.25 to $13.8 \mathrm{~ns}$ as the detection energy decreased from 1.91 to $1.55 \mathrm{eV}$. A recent PL decay study ${ }^{16}$ of low-nitrogen-content $a$-SiN $x$ :H films $(x<0.15)$ showed similar results. Three characteristic time constants were observed: $0.8,3.5$, and $14.0 \mathrm{~ns}$. The relative contribution to the decay from the shorter time constants increased with increasing nitrogen content, in agreement with results observed from HFCVD films.

\section{DISCUSSION}

Previous studies $9,11,13,15,35,37$ have identified two distinct peaks in the PL spectrum from amorphous silicon nitride films at energies of 3.1 (weak sharp band) and $2.5 \mathrm{eV}$ (strong broad band). Pundur et al. ${ }^{11}$ have observed two additional features in cathodoluminescence (CL) centered at 2.0 and $1.75 \mathrm{eV}$. They have proposed a model based on the $\mathrm{Si}-\mathrm{Si}$ $\left(\sigma-\sigma^{*}\right)$ bonds separated by $4.6 \mathrm{eV}$, representing the band edges, and additional electron and hole traps within the gap. According to their model, the $3.1 \pm 0.2 \mathrm{eV}$ transition occurs

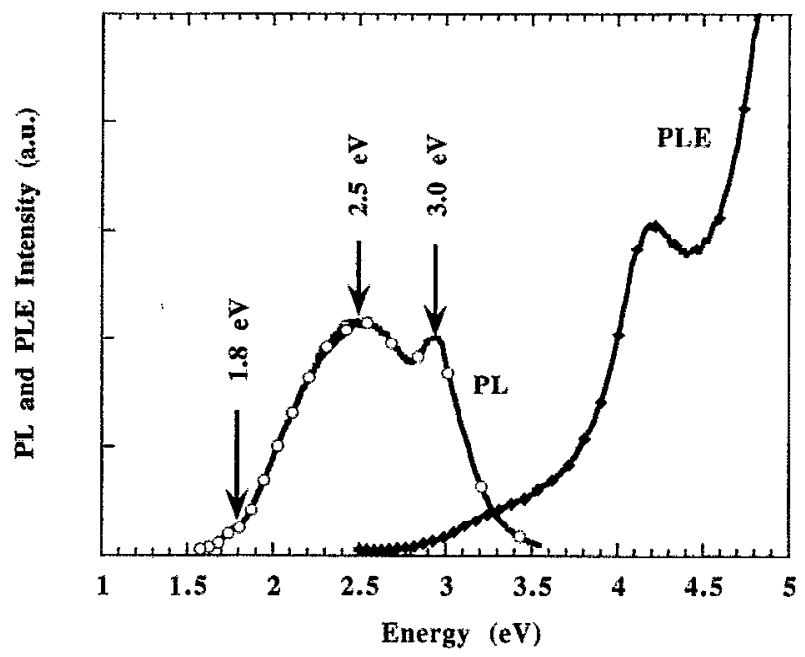

FIG. 7. PL and PLE in a film with larger contribution from the sharp $3.0 \mathrm{eV}$ peak. PL with excitation at $4.14 \mathrm{eV}$ and PLE with detection at $2.25 \mathrm{eV}$.

between an electron trap (with a trap depth between 1.3 and $1.5 \mathrm{eV}$ from the conduction band) and the valence band. The $1.75 \mathrm{eV}$ peak was observed by Vasilev et al. ${ }^{13}$ after hightemperature furnace annealing of stoichiometric $(\mathrm{N} / \mathrm{Si} \sim 1.33)$ silicon nitride films. This peak can also be explained by the same model. ${ }^{11}$ It is assigned to a radiative recombination between an electron and a hole in the traps giving rise to emission at $1.8 \mathrm{eV}(4.6-2 \times 1.4-1.8 \mathrm{eV})$. This was the only major mode of recombination observed in our silicon rich film (sample SQT5). As the band gap of this film only 2.43 $\mathrm{eV}$, the higher energy PL peaks cannot observed.

The main broad peak in the PL spectra was observed at an energy close to half of the optical band gap energy in all the samples we studied. Similar results have been obtained by other researchers for both silicon-rich ${ }^{9}$ and nitrogen-rich ${ }^{34}$ silicon nitride films. The main broad PL peak $(2.5 \pm 0.1 \mathrm{eV})$ arises from the radiative recombination between the electron and holes in the tails states of the conduction and valance bands and the metastable silicon dangling bond $\left(K^{0}\right)$ center.

Some of our samples have shown a larger contribution from the sharper $3.0 \mathrm{eV}$ peak as shown Fig. 7. This observation further confirms the existence of this level, as was observed recently by Savall et al. ${ }^{37}$ in their PECVD samples. Further work should be performed in order to correlate the relative contribution from the $3.0 \mathrm{eV}$ peak to the film deposition conditions and stoichiometry.

Robertson ${ }^{37}$ has defined defects in silicon nitride to be of four types, namely, $\mathrm{Si}-\mathrm{Si}$ and $\mathrm{N}-\mathrm{N}$ bonds, and $\mathrm{Si}$ and $\mathrm{N}$ dangling bonds. The author has calculated the $\mathrm{Si}-\mathrm{Si}$ bond to form a $\sigma$ state near the valence band and an empty $\sigma^{*}$ state close to the conduction band. The silicon dangling bond $\left(K^{0}\right.$ center) forms a gap state about the midgap. ${ }^{33,38}$ The $K^{0}$ center is known to be amphoteric and exists in both negatively and positively charged states. It is surmised ${ }^{34}$ that the silicon dangling bond, the $K^{0}$ center which forms a dominant trap and recombination center in silicon nitride, participates in the radiative transitions giving rise to the iuminescence. According to Robertson's theoretical predictions, there are two ni- 


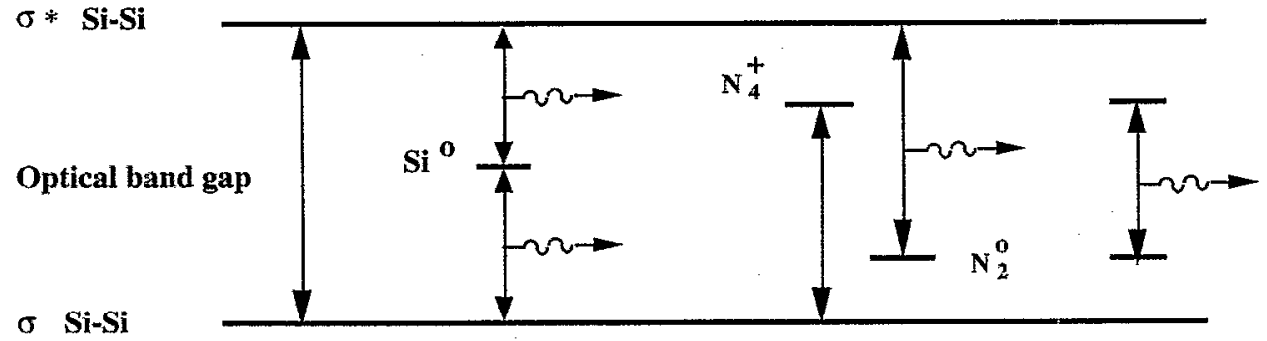

FIG. 8. Model for photoluminescence in $a-\mathrm{SiN}_{x}: \mathrm{H}$ films.

trogen defect states that give rise to levels within the gap, namely $\mathrm{N}_{4}^{+}$and $\mathrm{N}_{2}^{0}$, that have been calculated to be near the conduction and valence band, respectively. The presence of a nitrogen dangling bond defect has been confirmed by spin resonance measurements. ${ }^{31}$

Based on Robertson's work, ${ }^{38}$ a model shown in Fig. 8 may be proposed for the three peaks observed in our samples. These samples are amorphous and have varying optical band gaps (depending on the film composition), therefore, the energy levels are not very well defined. Furthermore, there is a distribution of states for a given defect giving rise to the broad features observed in the PL spectra. In any event, the three peaks observed in the luminescence spectrum of HFCVD films (see Figs. 5 and 7), can be explained by the proposed model. According to the model in Fig. 8, the $1.8 \mathrm{eV}$ peak is due to recombination between the $\mathrm{N}_{4}^{+}$and $\mathrm{N}_{2}^{0}$ levels. We attribute the broad $2.5 \pm 0.1 \mathrm{eV}$ peak to arise from recombination processes at the silicon dangling bond. Finally, the $3.0 \pm 0.1 \mathrm{eV}$ peak is due to recombination either from the conduction band to the $\mathrm{N}_{2}^{0}$ level or the valence band to the $\mathrm{N}_{4}^{+}$level. This model is in good agreement with the features observed in the luminescence spectra of the HFCVD silicon nitride films.

\section{CONCLUSIONS}

This article presents in great detail the optical properties of a series of silicon nitride films deposited by hot filament assisted chemical vapor deposition method. The optical band gap can be controlled by varying the film stoichiometry, which is a function of disilane flow rate. ESR measurements established that the films had a high density of silicon dangling bond centers, an order of magnitude greater than that observed from PECVD films. This was commensurate with a strong visible photoluminescence, which was also an order of magnitude greater than that observed from PECVD films. In addition to that two peaks were observed in the PL measurements of nitrogen-rich $\mathrm{a}-\mathrm{SiN}_{x}: \mathrm{H}$ films, and were attributed to a nitrogen dangling bond defect. These thermally stable, optically active defects in silicon nitride films have potential uses in visible emissive device applications, as demonstrated by the observation of weak electroluminescence from these films using a simple diode structure. In summary, this article presents a cohesive picture of optically active defects observed in HFCVD silicon nitride films and discusses their probable origin.

\section{ACKNOWLEDGMENTS}

The authors would like to thank Dr. Richard Sands for help with the electron spin resonance measurements. Assistance from Vinod Subramaniam in PL decay measurements is gratefully acknowledged. We have enjoyed useful discussions with Professor Jerzy Kanicki on properties of PECVD silicon nitride. The authors acknowledge support from NSF grant CTS 9301386, the NSF Center for Ultrafast Optical Science (STC PHY 8920108) at the University of Michigan, and the Center for Display Technology and Manufacturing at the University of Michigan.

${ }^{\prime}$ K. E. Bean, P. S. Gleim, and R. L. Yeakley, J. Electrochem. Soc. 114, 733 (1967).

${ }^{2}$ A. J. Lowe, M. J. Powell, and S. R. Elliot, J. Appl. Phys. 59, 1251 (1986).

${ }^{3}$ A. Piccirillo and A. L. Gobbi, J. Electrochem. Soc. 137, 3910 (1990),

${ }^{4}$ S. V. Hattangady, G. G. Fountain, R. A. Rudder, and R. J. Markunas, J. Vac. Sci. Technol. A 7, 570 (1989).

${ }^{5}$ D. V. Tsu and G. Lucovsky, J. Vac. Sci. Technol. A 4, 480 (1986).

${ }^{6} \mathrm{~K}$. A. Buckle, J. Rodgers, K. Pastor, C. Constantine, and D. Johnson, Appl. Phys, Lett. 60, 2601 (1992).

${ }^{7}$ S. V. Nguyen and K. Albaugh, J. Electrochem. Soc. 136, 2835 (1989).

${ }^{8}$ A. Markwitz, M. Bachmann, H. Baumann, K. Bethge, E. Krimmel, and P. Misaelides, Nucl. Instrum. Methods B 68, 218 (1992).

${ }^{9}$ I. G. Austin, W. A. Jackson, T. M. Searle, P. K. Bhat, and R. A. Gibson, Philos. Mag. B 52, 271 (1985).

${ }^{10}$ D. Chen, J. M. Viner, P. C. Taylor, and J. Kanicki, Mater. Res. Soc. Symp. Proc. 258, 661 (1992).

"P. A. Pundur, J. G. Shavalgin, and V. A. Gritsenko, Phys. Status Solidi A 94, K 107 (1986).

${ }^{12}$ V. V. Vasilev and I. P. Mikhailovikii, Phys. Status Solidi A 90, 355 (1985).

${ }^{13}$ V. V. Vasilev, I. P. Mikhailovskii, and K. K. Svitashev, Phys. Status Solidi A 95, K37 (1986).

${ }^{14}$ W. A. Jackson, T. M. Searle, I. G. Austin, and R. A. Gibson, J. Non-Cryst. Solids 77\&78, 909 (1985).

${ }^{15}$ V. V. Vasil'ev, I. P. Mikhailovskii, and K. K. Svitashev, Sov. Phys. Tech. Phys. 33, 510 (1988).

${ }^{16}$ C. Palsule, S, Gangopadhyay, S. Yi, J. M. Shen, H. A. Naseem, S. Kizzar, and F. H. C. Goh, Mater, Res. Soc. Symp. Proc, 284, 83 (1993).

${ }^{17}$ B. V. Spitsyn, L. L. Builov, and B. V. Deryagin, J. Cryst. Growth 52, 219 (1981).

${ }^{18}$ S. Matsumoto, Y. Sato, M. Kamo, and N. Setaka, Jpn. J. Appl. Phys. 21, L183 (1982).

${ }^{19}$ D. G. Goodwin and G. G. Gavillet, J. Appl. Phys. 68, 8329 (1991).

${ }^{20}$ R. R. Rye, J. Vac. Sci. Technol. A 9, 1099 (1991).

${ }^{21}$ J. L. Dupuie and E. Gulari, Appl. Phys. Lett. 59, 549 (1991).

${ }^{22} \mathrm{~S}$. V. Deshpande and E. Gulari, Mater. Res. Soc. Symp. Proc, 327, 115 (1994).

${ }^{23}$ S. V. Deshpande, E. Gulari, S. J. Harris, and A. M. Weiner, Appl. Phys. Lett. 65, 1757 (1994).

${ }^{24}$ K. Yasui, H. Katoh, K. Komaki, and S. Kaneda, Appl. Phys. Lett. 56, 898 (1990). 
${ }^{25} \mathrm{H}$. Matsumura, Jpn. J. Appl. Phys. 28, 2157 (1989).

${ }^{26} \mathrm{~J}$. L. Dupuie and E. Gulari, J. Vac. Sci. Technol. A 10, 18 (1992).

${ }^{27}$ S. V. Deshpande, J. L. Dupuie, and E. Gulari, Appl. Phys. Lett. 61, 1420 (1992).

${ }^{25}$ S. V. Deshpande, E. Gulari, S. W. Brown, and S. C. Rand, Mater. Res. Soc. Symp. Proc. 325, 177 (1994).

${ }^{29}$ J. Tauc, Amorphous and Liquid Semiconductors (Plenum, New York, 1974), p. 173.

${ }^{30}$ W. L. Warren, J. Kanicki, F. C. Rong, and E. H. Poindexter, J. Electrochem. Soc. 139, 880 (1992).

${ }^{31}$ W. L. Warren, P. M. Lenahan, and S. E. Curry, Phys. Rev, Lett. 65, 207 (1990).
${ }^{32}$ D. Jousse, J. Kanicki, D. T. Krick, and P. M. Lenahan, Appl. Phys. Lett. 52, 445 (1988).

${ }^{33}$ J. Robertson, Philos. Mag. B 63, 47 (1991).

${ }^{34}$ D. Chen, J. M. Viner, P. C. Taylor, and J. Kanicki, Mater. Res. Soc. Symp. Proc. 258, 661 (1992).

${ }^{35}$ V. A. Nadolinnyi, V. V. Vasilev, and I. P. Mikhailovskii, Phys. Status Solidi A 116, K105 (1989).

${ }^{36}$ W. Boonkosum, D. Kruangam, and S. Panyakeow, Jpn. J. Appl. Phys. 32, 1534 (1993).

${ }^{37}$ C. Savall, E. Bustarret, J. P. Stoquert, and J. C. Bruyere, Mater. Res. Soc. Symp. Proc. 284, 77 (1993).

${ }^{38}$ J. Robertson, Mater. Res. Soc. Symp. Proc. 284, 65 (1993). 